\title{
Mathematical model of parametrically controlled matched filters
}

Borislav Georgiev Naydenov ${ }^{1}$, Antim Hristov Yordanov $^{1}$ and Lyubomir Petrov Kamburov ${ }^{1}$

1 - Technical University of Varna, Department of Information and Communication Technologies, 9010, 1 Studentska Street, Varna, Bulgaria

Corresponding author contact: bornaeabv.bg

\begin{abstract}
One model of parametrically controlled coherent filters, with a wide application including radar systems, mobile communication systems to improve noise resistance, has been analysed. The application of the frequency domain-sampling theorem is used to obtain a set of frequency filters with variable parameters. These include autocorrelation signal processing, decomposition of Tailor series of the input signal, analysis of the control signal as a complex function with manageable parameters.
\end{abstract}

Keywords: radar systems, matched filters, noise resistance

\section{$1 \quad$ Introduction}

A one model of parametrically controlled coherent filters is described and analysed, applied in radar systems and mobile communication systems to improve noise resistance (Barton, 2004; Gray, 2006; Petrov, 2017). It is known that coherent filters, passive or active, in response to normal white noise, form an output response proportional to its autocorrelation function (Bissel, 2006; Fish, 2011).

$$
\left[S_{\text {out }}(t)\right]_{\text {coh }}=\operatorname{Re}\left\{\frac{A}{\pi} \int_{0}^{\infty} S_{\text {in }}{ }^{2}(\omega) e^{j \omega\left(t-t_{0}\right)} d \delta\right\}
$$

Where: $A=$ const with dimensionality $[A]=\frac{1}{\left[S_{\text {in }}(\omega)\right]}$;

$S_{i n}{ }^{t}$ и $(j \omega)=S_{i n} e^{\left\{j\left[\varphi_{s}(\omega)-\omega t_{d}\right]\right\}}$ and set the spectrum of the input signal with time delay $t_{d}$;

$t_{0}$ - moment of maximum of the autocorrelation function cover, $\omega$ - angular frequency.

In case of Gaussian noise,

$$
\left[S_{\text {out }}(t)\right]_{\text {coh }}=\operatorname{Re}\left\{\frac{A}{\pi} \int_{0}^{\infty}\left[S_{\text {in }}{ }^{2}(\omega) / w(\omega)\right] e^{j \omega\left(t-t_{0}\right)} d \omega\right\}=\operatorname{Re}\left\{\frac{A}{\pi} \int_{0}^{\infty} F^{2}(\omega) e^{j \omega\left(t-t_{0}\right)} d \omega\right\}
$$

Where:

$w(\omega)=W(\omega) / W_{0}$ - i.e. the normalized energy spectrum of the noise is reduced to the upper expression (1), the signal at the output of the parametric filter being equal to

$$
\left[S_{\text {out }}(t)\right]_{\text {prm }}=\operatorname{Re}\left\{\frac{A}{\pi} \int_{0}^{\infty} S_{\text {in }}(j \omega) e^{j \omega(t)}, \mathrm{H}(j \omega, t) d \omega\right\}
$$

Here:

$$
\mathrm{H}(j \omega, t)=\int_{0}^{\infty} h_{3}(y, t) e^{-j \omega(y)} d y
$$

$h_{3}(y, t)$ - is a pulse characteristic of the parametric system.

If we present $\mathrm{H}(j \omega, t)$ with the application of the theorem for signal sampling in the frequency domain (Marks, 2009), we will obtain the corresponding set of frequency filters with variable parameters. Again using the same theorem, but in the time domain we will get delays with deviations, each of which is a function of time (Ryzhak, 2003). In a number of cases, signal processing in a delayed-line filter model is practically unacceptable as large amount of deviations are required one after the other at extremely short intervals. Therefore, the model with a set of frequency filters, especially at the present 
level of development of microelectronics, is practically useful in micro-radar systems, (Proakis, 2000; Petrov, 2017).

\section{Explanation}

We assume that the input signal spectrum is limited by the ideal bandwidth filter in the range: $\omega_{0}-\omega_{s} / 2 \leq \omega \leq \omega_{0}+\omega_{s} / 2$.

Under this condition, we will present formula (1) in the following form:

$$
\begin{aligned}
& {\left[S_{\text {out }}(t)\right]_{\text {coh }}=\operatorname{Re}\left\{\frac{A}{\pi} \int_{\omega_{0}-\omega_{s} / 2}^{\omega_{0}+\omega_{s} / 2} S_{i n}^{2}(\omega) e^{j \omega\left(t-t_{0}\right)} d \omega\right\}=} \\
& =\operatorname{Re}\left\{\frac{A}{\pi} \sum_{i=1}^{N} \int_{\omega_{i}-\Delta \omega_{m} / 2}^{\omega_{i}+\Delta \omega_{m} / 2} S_{i n}^{2}\left[\omega_{i}+\left(\omega-\omega_{i}\right)\right] \times e^{\left\{j\left[\omega_{i}+\left(\omega-\omega_{i}\right)\right]\left(t-t_{0}\right)\right\}} d\left[\omega_{i}+\left(\omega-\omega_{i}\right)\right]\right\}
\end{aligned}
$$

Therefore:

$$
\begin{aligned}
& {\left[S_{\text {out }}(t)\right]_{\text {coh }}=\operatorname{Re}\left\{\frac{A}{\pi} \int_{\omega_{0}-\omega_{s} / 2}^{\omega_{0}+\omega_{s} / 2} S_{\text {in }}^{2}(\omega) e^{j \omega\left(t-t_{0}\right)} d \omega\right\}=} \\
& =\operatorname{Re}\left\{\frac{A}{\pi} \sum_{i=1}^{N} e^{j \omega_{i}\left(t-t_{0}\right)} \int_{-\Delta \omega_{m} / 2}^{\Delta \omega_{m} / 2} S_{i n}^{2}\left(\omega_{i}+\delta \omega\right) e^{j \delta \omega\left(t-t_{0}\right)} d \delta \omega\right\}
\end{aligned}
$$

Where:

$$
\left.\left.N=\omega_{s} / \Delta \omega_{m}\right\rangle\right\rangle 1 ; \omega_{i}=\omega_{0}-\omega_{s} / 2+\left(i-\frac{1}{2}\right) \Delta \omega_{m} ; i=1,2,3, \ldots, N .
$$

Breaking Taylor's order $S_{i n}{ }^{2}(\omega+\delta \omega)$, by degrees of $\delta \omega$ and limiting to his first member, we obtain:

$$
\left[S_{\text {out }}(t)\right]_{\text {coh }} \approx \operatorname{Re}\left\{\frac{A \Delta \omega_{m}}{\pi} \frac{\sin \frac{\Delta \omega_{m}}{2}\left(t-t_{0}\right)}{\frac{\Delta \omega_{m}}{2}\left(t-t_{0}\right)} \sum_{i=1}^{N} S_{\text {in }}^{2}\left(\omega_{i}\right) e^{j \omega_{i}\left(t-t_{0}\right)}\right\}
$$

Since $\Delta \omega_{m} t_{0}\langle\langle 1$, the above equation acquires the type:

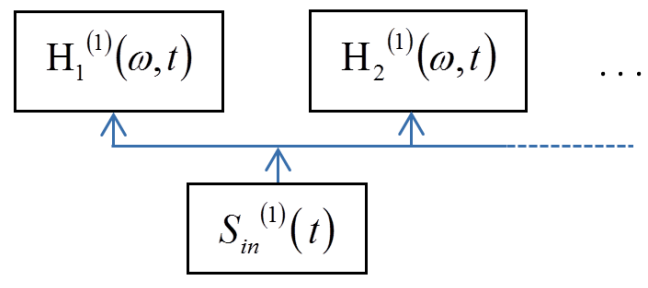

Fig. 1. Spectrum $S_{\text {in }}{ }^{(1)}(t)$ of signal transmitted to the controllable filters.

$$
\left[S_{\text {out }}(t)\right]_{\text {coh }} \cong \operatorname{Re}\left\{\frac{A \Delta \omega_{m}}{\pi} \sum_{i=1}^{N} S_{\text {in }}^{2}\left(\omega_{i}\right) e^{j \omega_{i}\left(t-t_{0}\right)}\right\}
$$

We convert the signal at the output of the parameter filter (3) in the same way (1), as the autocorrelation function response to the output signal from normal white noise disturbance, making the bandwidth of each of the frequency filter sets equal to $\Delta \omega_{m}$ (Naydenov, 2014).

With these assumptions, we will get:

$$
\left[S_{\text {out }}(t)\right]_{\text {prm }}=\operatorname{Re}\left\{\frac{1}{\pi} \int_{0}^{\infty} S_{\text {in }}(j \omega) e^{j \omega_{d}} \mathrm{H}(j \omega, t) e^{j \omega t} d \omega\right\} \approx \operatorname{Re}\left\{\frac{\Delta \omega_{m}}{\pi} \sum_{i=1}^{N} S_{\text {in }}\left(j \omega_{i}\right) e^{j \omega_{i}\left(t-t_{d}\right)} \mathrm{H}(j \omega, t)\right\}
$$

Where, in the case of a periodic increase of each of the frequency filters with frequency $\omega_{i}$ the member $\mathrm{H}(j \omega, t)$ will be, fig. 1 : 


$$
\mathrm{H}\left(j \omega_{i}, t\right)=\mathrm{H}_{0}\left(j \omega_{i}\right)+\mathrm{H}_{1}\left(j \omega_{i}\right) \cos \left(\omega_{i} t+\chi_{1 i}\right)+\mathrm{H}_{2}\left(j \omega_{i}\right) \cos \left(2 \omega_{i} t+\chi_{2 i}\right)+\ldots
$$

Since the spectrum $\left[S_{\text {out }}(t)\right]_{\text {coh }}$ is locked in the range $\omega_{0}-\omega_{s} / 2 \leq \omega \leq \omega_{0}+\omega_{s} / 2$, the upper row (10) of this requirement only corresponds to two members $\mathrm{H}_{0}\left(j \omega_{i}\right)$ and $\mathrm{H}_{2}\left(j \omega_{i}\right) \cos \left(2 \omega_{i} t+\chi_{2 i}\right)$. It $\mathrm{H}_{0}\left(j \omega_{i}\right)$ does not provide any options compared to passive matching filters. Interesting is the member $\mathrm{H}_{2}\left(j \omega_{i}\right) \cos \left(2 \omega_{i} t+\chi_{2 i}\right)$ that converts the frequency $2 \omega_{i}-\omega_{i}=\omega_{i}$. If we substitute $\mathrm{H}_{0}\left(j \omega_{i}\right)(10)$ in an expression (9) and separate from the spectrum $\left[S_{\text {out }}(t)\right]_{p r m}$ a frequency corresponding to the combination $2 \omega_{i}-\omega_{i}$, we get an output signal that must be proportional to $\left[S_{\text {out }}(t)\right]_{\text {coh }}$ (Ryzhak, 2003). As a result, we will obtain a system of equations defining the required characteristics of a manageable coherent filter:

$$
\sum_{i=1}^{N} S_{i n}\left(\omega_{i}\right) \mathrm{H}_{2}\left(\omega_{i}\right) \cos \left[\omega_{i} t+\omega_{i} t_{d}-\varphi_{s}\left(\omega_{i}\right)+\chi_{2 i}\right]=\beta A \sum_{i=1}^{N} S_{i n}{ }^{2}\left(\omega_{i}\right) \cos \omega_{i}\left(t-t_{0}\right)
$$

Or if, we equate to zero:

$$
\sum_{i=1}^{N} S_{i n}\left(\omega_{i}\right) \mathrm{H}_{2}\left(\omega_{i}\right) \cos \left[\omega_{i} t+\omega_{i} t_{d}-\varphi_{s}\left(\omega_{i}\right)+\chi_{2 i}\right]-\beta A \sum_{i=1}^{N} S_{i n}{ }^{2}\left(\omega_{i}\right) \cos \omega_{i}\left(t-t_{0}\right)=0
$$

We will obtain:

$$
\sum_{i=1}^{N} \mathrm{H}_{2}\left(\omega_{i}\right) \cos \left[\omega_{i}\left(t-t_{0}\right)+\omega_{i}\left(t_{0}+t_{d}\right)-\varphi_{s}\left(\omega_{i}\right)+\chi_{2 i}\right]-\beta A S_{i n}\left(\omega_{i}\right) \cos \omega_{i}\left(t-t_{0}\right)=0
$$

Equation (13) is satisfied when:

$$
\begin{aligned}
& \omega_{i}\left(t_{0}+t_{d}\right)-\varphi_{s}\left(\omega_{i}\right)+\chi_{2 i}=2 \pi l, l=0,1, \ldots, \\
& \mathrm{H}_{2}\left(\omega_{i}\right)=\beta A S_{i n}\left(\omega_{i}\right), \text { for each } i=1,2,, \ldots, N
\end{aligned}
$$

To find a clear dependency of $\mathrm{H}\left(j \omega_{i}, t\right)$ from a control signal $v_{\text {contr. } i}$, we will look $\mathrm{H}\left(j \omega_{i}, t\right)$ at a complex function with controllable parameters $\zeta_{i}$ dependent on control signals, $v_{c o n t r . i}$, that is

$$
\mathrm{H}\left[j \omega_{i}, \varsigma_{i}\left(v_{\text {contr. } i}\right)\right]
$$

Breaking (16) in Taylor's order by degrees of $v_{\text {contr.i }}$ / note: the control signal will be recorded without the "contr." Index/, we obtain:

$$
\begin{aligned}
& \mathrm{H}\left[j \omega_{i}, \varsigma_{i}\left(v_{i}\right)\right]=\mathrm{H}\left[j \omega_{i}, \varsigma_{i}(0)\right]+\frac{d \mathrm{H}\left[j \omega_{i}, \varsigma_{i}(0)\right]}{d \varsigma_{i}} \frac{d \varsigma_{i}(0)}{d v_{i}} v_{i}+ \\
& +\frac{1}{2 !}\left[\frac{d \mathrm{H}\left[j \omega_{i}, \varsigma_{i}(0)\right]}{d \varsigma_{i}} \frac{d^{2} \varsigma_{i}(0)}{d v_{i}{ }^{2}}+\frac{d^{2} \mathrm{H}\left[j \omega_{i}, \varsigma_{i}(0)\right]}{d \varsigma_{i}{ }^{2}}\left(\frac{d \varsigma_{i}(0)}{d v_{i}}\right)^{2}\right] v_{i}^{2}+\ldots
\end{aligned}
$$

Note that this $v_{i}$ is a function of time:

$$
\begin{aligned}
& v_{i}(t)=\operatorname{Re}\left\{\frac{1}{\pi} \int_{\omega_{i}-\Delta \omega_{m} / 2}^{\omega_{i}+\Delta \omega_{m} / 2} S_{\text {contr. }}(\omega) e^{j \varphi_{\text {contr. }}(\omega)} e^{-j \omega_{\text {contr. }} .} \times e^{-j \omega t} d \omega\right\} \approx \\
& \approx \frac{1}{\pi} S_{\text {contr. }}\left(\omega_{i}\right) \Delta \omega_{m} \cos \left[\omega_{i}\left(t-t_{\text {contr. }}\right)+\varphi_{\text {contr. }}\left(\omega_{i}\right)\right]
\end{aligned}
$$

Where:

$S_{\text {contr. }}(j \omega)=S_{\text {contr. }}(\omega) e^{j \varphi_{\text {contr. }}(\omega)}$ - is the spectrum of the control signal; $t_{\text {contr. }}$ - the moment of control signal input; $\varphi_{\text {contr. }}$ - is the phase offset. It is clear from the expressions (17 and 18) that the quadratic element of the order (17) generates the second harmonic of $v_{i}$. We ignore the influence of the higher harmonic members in the second member, due to their small values (Ryzhak, 2003). 
It has to be said that if we consider all the filters to be identical and count $\omega_{i}$ as their resonance frequency at $v_{i}=0$, then we will obtain:

$$
\mathrm{H}_{2}\left(\omega_{i}\right) \cos \left(2 \omega_{i} t+\chi_{2 i}\right)=\frac{\Delta \omega_{m}{ }^{2} \varsigma_{2 i}}{2 \pi^{2}} S_{\text {contr. }}^{2}\left(\omega_{i}\right) \cos \left[\omega_{i}\left(t-t_{\text {contr. }}\right)+\varphi_{\text {contr. }}\left(\omega_{i}\right)\right]
$$

$\varsigma_{2 i}$ is a coefficient, in front of $v_{i}{ }^{2}$ of the row (17).

\section{Conclusion}

If we substitute the expression (19) in the expressions (14) and (15) we will obtain:

$\omega_{i}\left[t_{0}-\left(2 t_{\text {contr. }}-t_{d}\right)\right]+2 \varphi_{\text {contr. }}\left(\omega_{i}\right)-\varphi_{s}\left(\omega_{i}\right)=0$, for $i=1,2, \ldots, N ; l=0$

Then:

$$
\begin{gathered}
t_{0}=2 t_{\text {contr. }}-t_{d}=t_{\text {contr. }}+\left(t_{\text {contr. }}-t_{d}\right), t_{d} \text { and } t_{0} \text { are symmetrical about } t_{\text {contr. }} \\
\varphi_{\text {contr. }}\left(\omega_{i}\right)=\frac{1}{2} \varphi_{s}\left(\omega_{i}\right) \quad i=1,2, \ldots, N ; l=0
\end{gathered}
$$

$\frac{\Delta \omega_{m}{ }^{2} S_{2 i}}{2 \pi^{2}} S_{\text {contr. }}^{2}\left(\omega_{i}\right)=\beta_{i} A S_{\text {in }}\left(\omega_{i}\right)$, from where

$$
S_{\text {contr. }}\left(\omega_{i}\right)=B\left[S_{\text {in }}\left(\omega_{i}\right)\right]^{\frac{1}{2}}, \quad i=1,2, \ldots, N
$$

or $B=\left[\frac{2 \pi^{2} \beta A}{\varsigma_{2 i} \Delta \omega_{m}{ }^{2}}\right]^{\frac{1}{2}}$.

If at the moment $t_{0}$, we want to limit the spectrum $S_{i n}(j \omega)$ of the signal passed to the controlled filter input - fig. 3 , it is necessary to submit to it the above-described control signals satisfying the relations (20), (21), (22).) The condition of the expression (21) mean that, at an input signal with a linear law of variation of the frequency modulation steepness, the law of the frequency modulated control signal should be twice as large fig. 2 (Ryzhak, 2003; Cook, 1971; Naydenov, 2014).

In the examined model of a controlled matched filter, each of its sectors - Fig. 2 is essentially a singlecontour parameter converter. The model can be summarized for a case of a two-loop parameter converter

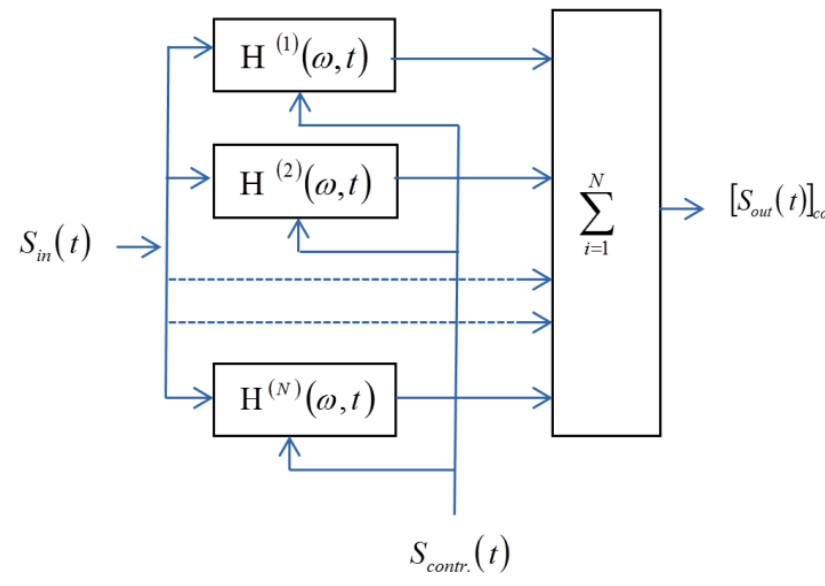

Fig. 2 Block diagram of a parametrically controlled filter. in each of the controllable filter sectors. It should also be said that the software simulation

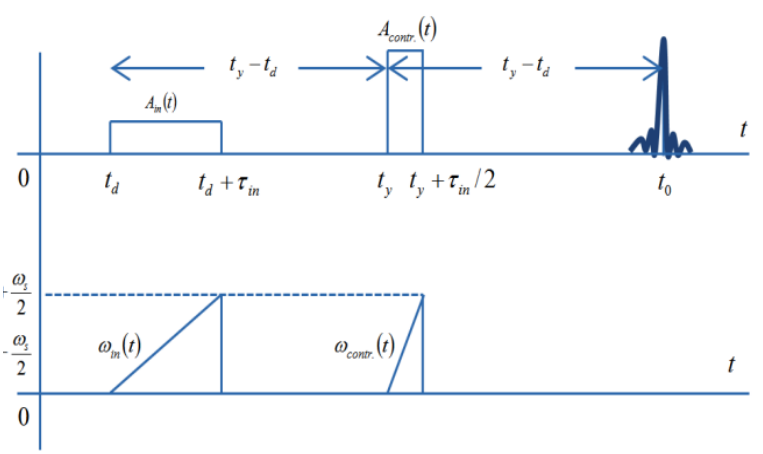

Fig. 3. Spectral characteristics of a controlled coherent filter.

could examine the possibilities of sampling the control signal and determining the spectral characteristic of the output signal. The analysis of variations of frequency and temporary filter parameters within certain limits opens the possibility of examining the purity of the spectral characteristics of the output signal. 


\section{References}

Ryzhak, I.S. (2003). Selected problems of the theory of circuits and signal processing. Russian. Publisher: Blok-Inform-Experss. link.springer.com/article/10.1134/S1064226914090071

Bissell, C. C. and V. E. Katsnelson (2006), (Trans.), accessible at http://ict.open.ac.uk/classics/ or via the Open University Open Research", Online at http://oro.open.ac.uk indexed as Bissell Classic Papers in Information \& Communication Technology.

Petrov, P. P., B. G. Naydenov, G. C. Chervenkov. (2017). Radiocommunication technic. Technical university, Varna, Bulgaria, ISBN978-619-7349-01-6.

Marks, R.J.(II). (2009) Handbook of Fourier Analysis and Its Applications, Oxford University Press http://www.twirpx.com/file/1422794/

Proakis John. Digital connections. /translation/ Under the line. D.D. Klovskogo. (2000) - M. Radio and Sighs. https://www.scribd.com/doc/6659361/pdf-DSP-Real-Time-Digital-Signal-

Processing

Cook C. E., Bernfeld M. (1967) Radar Signals, Academic Press, New York. Radiolocation signals. Theory and application. - M.: Soviet Radio 1971. /translation/ https://www.twirpx.com/file/2296229/

Naydenov, B. G., A. H. Yordanov. (2014) Analysis of the possibilities of filtering signals by predicting errors with linear invariant time discrete systems - Science Session -2014. National Military University "Vasil Levski", Faculty of Artillery Air Defense and Information Security. Shumen, Bulgaria.

Barton David K. (2004) Radar System Analysis and Modeling. https://www.twirpx.com/file/1813015/

Fish, A., Gurevich, S.; Hadani, R.; Sayeed, A.; Schwartz, O. (2011) Computing the matched filter in linear time". arXiv:1112.4883.

Gray, D.A. (2006) Multi-channel Noise Radar, Proc. Of International Radar Symposium , IRS 2006, Krakow, Poland. 